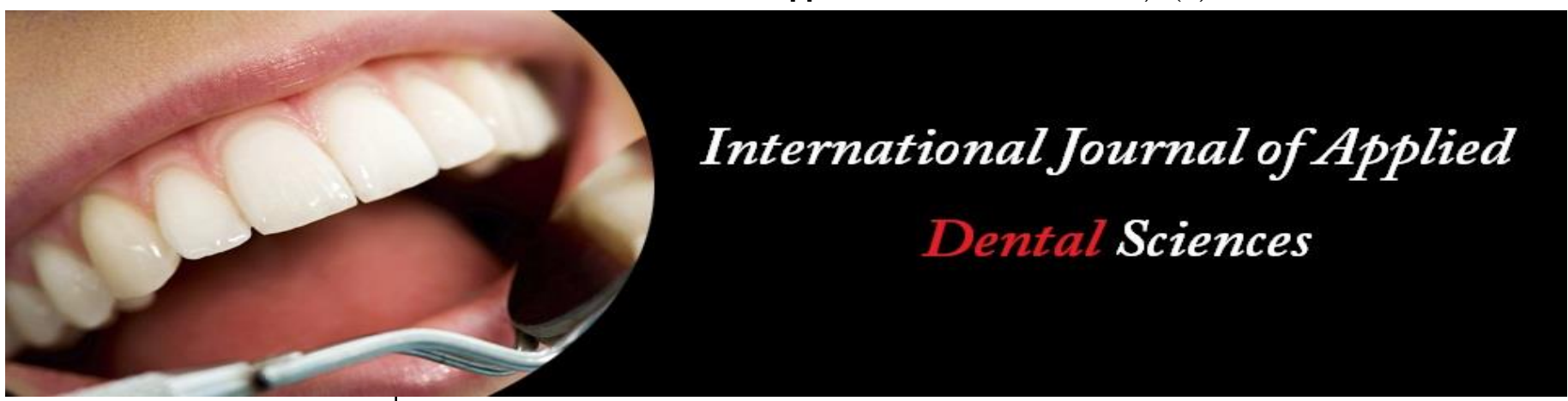

ISSN Print: 2394-7489

ISSN Online: 2394-7497

IJADS 2021; 7(2): 423-427

(C) 2021 IJADS

www.oraljournal.com

Received: 01-02-2021

Accepted: 03-03-2021

Dr. Rary Rajan Panackal

Post-Graduate, Department of

Prosthodontics Crown and

Bridge, KVG Dental College,

Sullia, Karnataka, India

Dr. Suhas Rao K

HOD, Department of

Prosthodontics Crown and

Bridge, KVG Dental College,

Sullia, Karnataka, India
Corresponding Author: Dr. Rary Rajan Panackal Post-Graduate, Department of Prosthodontics Crown and Bridge, KVG Dental College, Sullia, Karnataka, India

\section{Correlation between inter inner canthal distance and occlusal vertical dimension among young adults of Dakshina Kannada population: An in vivo study}

\author{
Dr. Rary Rajan Panackal and Dr. Suhas Rao K
}

DOI: https://doi.org/10.22271/oral.2021.v7.i2g.1240

\section{Abstract}

Objective methods of determining the vertical dimension of occlusion offer significant advantages because no radiographs or others special measuring devices are required. One of the objective measurement of vertical dimension of occlusion is twice the intercanthal distance. Hence this study was aimed to evaluate the correlation between the length of vertical dimension of occlusion and the length of twice the inter inner canthal distance among Dakshina Kannada population. Innercanthal and occlusal vertical dimension measurements were taken using digital vernier caliper from 60 subjects that fulfilled all criteria stated. The results were statistically analyzed. There was positive correlation between twice inner canthal distance and occlusal vertical dimension in Dakshina kannada population.

Keywords: inner canthal distance, occlusal vertical dimension, Dakshina Kannada population

\section{Introduction}

A successfully accomplished vertical dimension is always of chief significance in the management of patients undergoing oral rehabilitation.

Even though the core of Prosthodontics has advanced to fixed prostheses, still the concepts like vertical jaw relation provide foundation for all the prosthetic rehabilitation procedures ${ }^{[1]}$.

The vertical dimension is defined as "the distance between two selected anatomical or marked points (generally one on the tip of the nose and the other on the chin), one on a fixed member and the other on a mobile member ${ }^{[2]}$. (GPT-9)

Various techniques of determining the vertical dimension has been introduced, but there is no scientific method that can be said accurate for all individual. Techniques of determining the vertical dimension in general are broadly classified into mechanical method and physiological $\operatorname{method}^{[3]}$.

The mechanical methods such as jaw relation methods and recording pre-extraction methods that are further classified into three methods: radiographic profile, study casts, and facial measurements ${ }^{[3]}$. The physiological methods include the measurement of the physiologic rest position, phonetics, swallowing phenomenon, and tactile sense ${ }^{[3]}$.

The development of methods for determining the correct distance remains a controversial topic in the literature. Most of them are not scientifically accurate and none seem to be considered superior to the others ${ }^{[4]}$.

The inner canthal distance (ICD) is defined as the distance between the medial angles of the palpebral fissures bilaterally. It was reported that in $78 \%$ of the population evaluated, the inner inter canthal distance was attained by the time the subject was one year old. Subsequently, the growth rate in this area becomes slow in contrast to the outer orbital dimensions. At 5 years of age, $93 \%$ of inner canthal distance growth is achieved; maturity is reached between 8-11 years. The inner canthal distance of 28 to $35 \mathrm{~mm}$ is considered to be normal irrespective of sex, race and age ${ }^{[5]}$.

The purpose of this study is to evaluate the correlation between the length of vertical dimension of occlusion (VDO) and the length of twice inter inner canthal distance (ICD) among Dakshina Kannada population. 


\section{Materials and Methods}

The study was conducted in Department of Prosthodontics including Crown and Bridge and Implantology in K.V.G. Dental College and Hospital, Sullia, DK. A total of 60 subjects (30 males and 30 females) with angles class 1 molar relationship, pleasing profile and sound morphologically normal permanent dentition were included in the study. All subjects' ranged between 18-30 years in age and was native of Dakshina Karnataka with no obvious facial deformities. Patients with posterior bite collapse as a result of loss of teeth and subjects having excessive amount of soft tissues under the chin were excluded. Subjects with increased lower facial height, previous or currently undergoing orthodontic treatment, presence of tempromandibular joint disorders, aberration of the cornea (microcornea, macrocornea, congenital anomalies, aniridia, iris atrophy, iris cyst) were not included in the study.

After selecting the subject suitable for the study, procedure was explained to them and after obtaining their consent; measurements were taken on fully dentate patients when teeth were in contact sitting in an upright position looking forward, head without any support and occlusal plane parallel to the floor. Digital Vernier Caliper was used to measure the occlusal vertical dimension and inner canthal distance.

The facial width will be measured according to two categories:

1. Inner Inter-canthal width is defined as the distance between the right medial canthus and the left medial canthus.

2. Vertical dimension of occlusion: the distance between two selected anatomic or marked points when in maximal intercuspal position.

\subsection{Recording facial measurement}

\subsubsection{Measurement of inner inter-canthal distance}

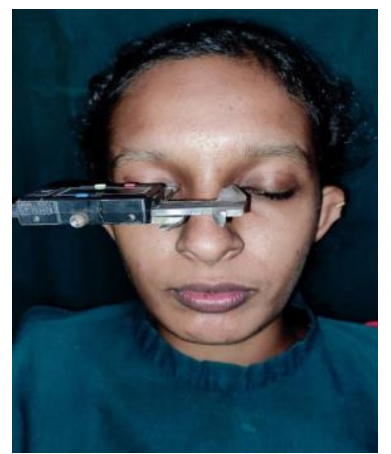

Fig 1: Measurement of inner inter-canthal distance

Subjects were seated on a dental chair with their head supported in an upright position so that they look forward. A digital vernier caliper was placed against their forehead and then lowered towards their eyes. The arms of the caliper were adjusted carefully so that they are in gentle contact with the median angle of the palpebral fissures of both the eyes (Fig:
1). The value on the digital verniercaliper was then recorded. The distance between the two anatomical landmarks was recorded as the inner inter canthal distance. In order to avoid bias, care was taken to measure accurately by repeating the readings three times and the average was recorded for all measurements.

\subsubsection{Measurement of the vertical dimension of occlusion}

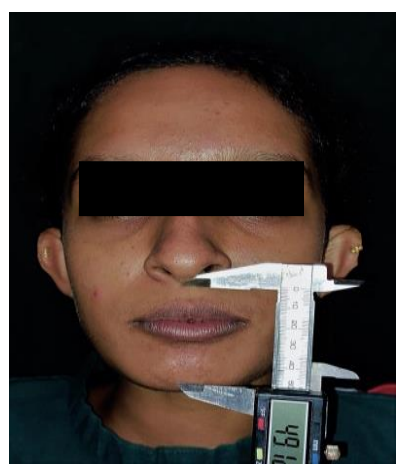

Fig 2: Measurement of the vertical dimension of occlusion

Subjects were seated on a dental chair with their head in an upright position. A digital vernier caliper was placed such that the beaks of the instrument are at their contact point with the subnasion and gnathion. Measurements were made in a straight line (Fig: 2). The value on the digital vernier caliper was recorded. In order to avoid bias, care was taken to measure accurately by repeating the readings three times and the average was recorded for all measurement.

A pilot study was conducted among 15 participants to determine the correlation between ICD and VDO. Analysis revealed a correlation coefficient of 0.7 which was strong correlation.

Sample size was calculated using correlation coefficient obtained, using the formula:

$$
\mathrm{N}=\frac{\left(\underline{\mathrm{z}}_{1-\alpha /(2 \tau)}+\mathrm{z}_{1-\beta}\right)^{2}+3}{[\mathrm{c}(\mathrm{r})]^{2}}
$$

Where $c(r)=1 / 2 \ln [1+r / 1-r]$

$\mathrm{z} 1-\alpha /(2 \tau)=1.96$ for $95 \%$ confidence interval

$z 1-\beta=0.84$ for $80 \%$ power

$\mathrm{r}=$ anticipated correlation coefficient

\section{Results}

The measurements were obtained from the patient by one independent observer and average value was tabulated and analyzed statistically. All measurements were obtained in millimeters.

The database of all study sample measurements was analyzed in SPSS version 20. The results were tabulated using "Pearson's correlation and Student t- test" and probability (p) value for each parameter was calculated.

\begin{tabular}{|c|c|c|c|c|c|}
\hline \multicolumn{6}{|c|}{ Group statistics } \\
\hline & Gender & $\mathbf{N}$ & Mean & Std. deviation & Std. error mean \\
\hline \multirow{2}{*}{ ICD } & Male & 30 & 29.1108 & 1.89816 & .34655 \\
\hline & Female & 30 & 27.5870 & 1.71322 & .31814 \\
\hline \multirow{2}{*}{ OVD } & Male & 30 & 60.2123 & 4.09334 & .74734 \\
\hline & Female & 30 & 57.2461 & 5.46454 & 1.01474 \\
\hline \multirow{2}{*}{ Twice ICD } & Male & 30 & 58.2216 & 3.79632 & .69311 \\
\hline & Female & 30 & 55.1740 & 3.42644 & .63627 \\
\hline
\end{tabular}

Table 1: Measurements of inner canthal distance, occlusal vertical dimension and twice inner canthal distance among study participants $(\mathrm{n}=60)$ 
Table 1 indicates the results of current study. The mean length of occlusal vertical dimension was recorded $58.72 \mathrm{~mm}$ while the mean length of twice ICD was $56.69 \mathrm{~mm}$. The mean values for length of occlusal vertical dimension were found to be
$60.21 \mathrm{~mm}$ among males and 57.24 among females. The mean length of twice ICD was found to be $58.22 \mathrm{~mm}$ among males and $55.17 \mathrm{~mm}$ among females.

Table 2: Correlation between vertical dimension and twice inner canthal distance $(n=60)$

\begin{tabular}{|c|c|c|c|}
\hline \multicolumn{4}{|c|}{ Correlations } \\
\hline \multirow{2}{*}{ OVD } & Pearson Correlation & VD & Twice ICD \\
\hline \multirow{3}{*}{ Twice ICD } & Sig. (2-tailed) & 1 & $.532^{* *}$ \\
\cline { 2 - 4 } & $\mathrm{N}$ & 59 & .000 \\
\cline { 2 - 4 } & Pearson Correlation & $.532^{* *}$ & 59 \\
\cline { 2 - 4 } & Sig. (2-tailed) & .000 & 1 \\
\cline { 2 - 4 } & $\mathrm{N}$ & 59 & 59 \\
\hline
\end{tabular}

**Correlation is significant at the 0.01 level (2-tailed).

Pearson correlation indicates that there is a statistically significant positive correlation between occlusal vertical dimension and twice inner canthal distance (Table 2).

Table 3: Comparison of inner canthal distance and occlusal vertical dimension between males and females among Dakshinna Kannada population $(\mathrm{n}=30)$

\begin{tabular}{|c|c|c|c|c|c|c|c|}
\hline & \multicolumn{9}{|c|}{ t-test for equality of means } \\
\cline { 2 - 8 } & $\mathbf{t}$ & \multirow{2}{*}{$\mathbf{d f}$} & \multirow{2}{*}{ Sig. (2-tailed) } & $\begin{array}{c}\text { Mean } \\
\text { difference }\end{array}$ & $\begin{array}{c}\text { Std. error } \\
\text { difference }\end{array}$ & \multicolumn{2}{|c|}{ 95\% Confidence interval of the difference } \\
\cline { 5 - 9 } & & 57 & .002 & 1.52377 & .47127 & .58007 & Upper \\
\hline ICD $\times 2$ & 3.233 & 57 & .021 & 2.96624 & 1.25415 & .45486 & 5.46746 \\
\hline OVD & 2.365 & 57 & &
\end{tabular}

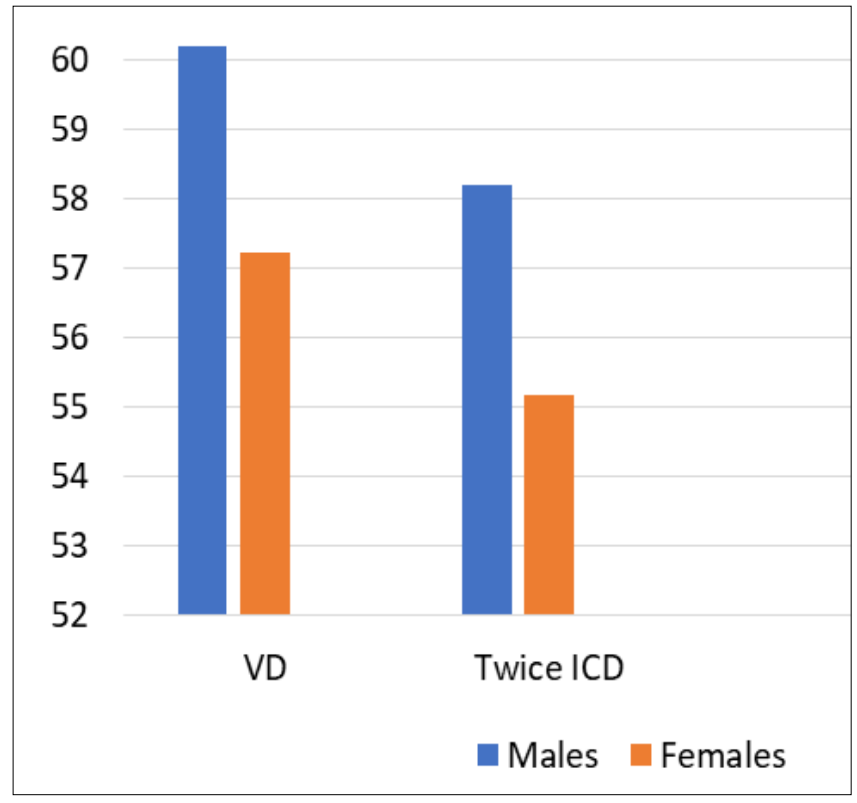

Graph 1: Comparison of inner canthal distance and occlusal vertical dimension between males and females among Dakshinna Kannada population $(\mathrm{n}=30)$

From the $\mathrm{T}$ test it can be concluded that there is a statistically significant difference between twice ICD and OVD among males and females (Table 3). The OVD and ICD were significantly higher for males than for females (Graph 1) among Dakshina Kannada population.

\section{Discussion}

Losing teeth and procuring an artificial prosthesis is not a pleasant experience for any individual. However, the distress of the patient can be lowered by providing a prosthesis which restores the original facial appearance and functions similar to natural teeth. Positively, establishing a correct vertical dimension of face is one of the important factors to be considered in attaining this objective. Literature review depicted that many methods have been described over the years for determination of vertical dimension, but none of them is fully accepted or considered completely correct ${ }^{[1]}$. The greatest risk in the phase of denture construction is an excessive inter-arch distance because premature striking of teeth causes recurring trauma on the tissues and longer leverage, making the denture more difficult to manipulate and displaced easily. This defective occlusal contact may result in clicking of the dentures ${ }^{[9]}$.

Methods to establish the occlusal vertical dimension can either be subjective or objective. The subjective methods constitute evaluation of esthetics, phonetics, swallowing and patient comfort. The objective methods include electromyographical records, biting power and the utilization of facial measurement. The traditional methods comprising the judgment of facial esthetics and patient comfort sounds well subjectively but are too nonspecific scientifically. Tooth display, lip support, harmonious relationships, and facial pictures do not substitute for those patients without accurate record. The objective methods like electromyography and biting forces are impractical as they demand the use of complex devices and cannot be routinely used. There is still no accurate method to elicit the original position of upper anterior teeth in edentulous patient. Hence, the use of facial dimensions for establishing the Occlusal Vertical Dimension can be considered to be more practical objectively and subjectively ${ }^{[12]}$. The present investigation was therefore undertaken to find a simple yet feasible method by studying the relationship between occlusal vertical dimension and twice length of innercanthal distance.

Inner inter canthal distance was selected for measurement in the present study since it has been reported to be a stable anthropometric parameter ${ }^{[16]}$; the reference points (namely, medial angles of the palpebral fissures of the eyes) can be identified and located effortlessly; and these reference points can be measured simply with an instrument such as a Vernier caliper. 
Relevance of facial measurements in dentistry was first mentioned by Ivy ${ }^{[12]}$. The mandible is an ever changing and a mobile member of the cranium, any landmark or measurement which does not involve the mandible will minimize errors in recording. McGee ${ }^{[13]}$ and Pound ${ }^{[14]}$ in their study considered vertical dimension to be the distance between subnasion and gnathion ${ }^{[6]}$.

Numerous studies have shown high variability in biometric parameters among the individuals of different populations, races, and countries. Thus, before making any concept universal, widespread studies are required among different populations to find the existence of any specific correlation. No definitive guidelines twice ICD and OVD exist. Hence, in the present study in order to find the correlation between twice inner intercanthal distance and occlusal vertical dimension, subjects were selected from Dakshina Kannada population, Karnataka. A total of 60 subjects in the age group of 18-30 years with equal number of males and females were evaluated.

The results of the study showed that there is significant positive correlation between twice ICD and to length of OVD. This finding is consistent with the findings of Misch ${ }^{[11]}$ in which he stated that the original VDO was most often similar to at least 12 other dimensions on the face and hands and may be objectively determined in most patients. One of the dimensions is twice the distance between the inner canthus of both eyes ${ }^{[7]}$. The research by Misch ${ }^{[11]}$, were done in United States which is mostly represented by Caucasian people who belong to the Caucasoid race. According to Misch ${ }^{[11]}$, the original VDO is most often similar to twice the distance between the inner canthus of both eyes. On the contrary, this study was conducted among Dakshina Kannada population. In a study by Majeed et al. ${ }^{[1]}$ the mean value for two times of inner canthus of right eye to inner canthus of left eye was $62.29 \mathrm{~mm}$. The mean value of parameter two times of inner canthus of right eye to inner canthus of left eye coincided with the chin-nose distance. Therefore, Majeed et al. [1] proposed twice ICD can be used for the determination of OVD among Pakistani individuals.

The results showed that there were significant differences in the mean length of VDO and twice the ICD between male and female samples (Table 3 and Graph1). This finding is consistent with the research done by Oladipo et al. ${ }^{[15]}$. In his research, he revealed that the mean values for all the parameters of facial measurements were significantly higher in male than in female subjects $(P<0.05)$, with exception of nasal height. This difference can be because of facial dimensions were modified during growth and development with sex-related characteristics. Individual variation across male and female include a range of features; for example, clear differences are found in the length and width of the nose and mouth, the inclinations of forehead and nose, as well as the overall shape of the face ${ }^{[7]}$.

The results obtained in this research support the hypothesis, hence the length of twice inner canthal distance can be suggested to be used as one of the guide in the determination of OVD. However, other method of determination of OVD should be used together in order to obtain a more accurate measurement.

To validate these findings, further studies should be carried out that would include the similar analysis for dentulous population in other ethnic groups which can be accepted universally. However, the operator should be aware that OVD is the result of a mucoskeletal balance. The correct OVD can be described well as a range instead of as a fixed point.
Therefore, in order to assess the OVD, a pluralistic method should be embraced at all the stages of rehabilitation in order to maximize the benefits and minimize damage to the stomatognathic system ${ }^{[9]}$.

The limitation of this study was that it was restricted to the subjects with Class I malocclusion and other skeletal or dental malocclusions were not considered. Also, the subjects were not categorized based on facial forms. Further, the measurements are difficult to record when patient has a round facial profile with excessive soft tissue bulk under the chin ${ }^{[9]}$.

\section{Conclusion}

Within the limitations of this study, it can be concluded that

- There was no significant difference between the mean length of VDO and twice the ICD. Therefore, the length of twice the ICD can be recommended to be used clinically as one of the guide in the determination of OVD when the patient's OVD is already lost in this population. However, other methods of determination of OVD should also be used in order to obtain a more accurate measurement.

- The OVD and twice ICD are significantly higher for males than for females in Dakshina Kannada population. Despite of racial differences and sexual dimorphism between males and females, a proportional relationship exists between the length of OVD and the length of twice ICD.

\section{References}

1. Majeed MI, Afzal M, Kashif M. Determination of occlusal vertical dimension in a section of Pakistani population using craniofacial measurements. Journal of University Medical \& Dental College 2015;6(1):1-5.

2. Ferro KJ, Morgano SM, Driscoll CF, Freilich MA, Guckes AD, Knoernschild KL et al. The Glossary of Prosthodontic Terms.

3. Miftahullaila M, Primasari A. The Proportion of Proto Malayan's and Deutro Malayan's Vertical Dimension Using Willis's Method, Mc. Gee's Method and Golden Proportion Concept Among Students of Faculty of Denstistry In University of Sumatera Utara.

4. Discacciati JA, de Souza EL, Vasconcellos WA, Costa SC, de Magalhães Barros V. Increased vertical dimension of occlusion: signs, symptoms, diagnosis, treatment and options. The Journal of Contemporary Dental Practice 2013;14(1):123.

5. Wazzan KA. The relationship between intercanthal dimension and the width of maxillary anterior teeth. J Prosthet. Dent 2001;86(6):608-611.

6. Brar A, Mattoo KA, Singh Y, Singh M, Khurana PR, Singh M. Clinical reliability of different facial measurements in determining vertical dimension of occlusion in dentulous and edentulous subjects. International Journal of Prosthodontics \& Restorative Dentistry 2014;4(3):68.

7. Yaw LK, Subrata G, Ardan R. Determination of vertical dimension using intercanthal distance in adult Mongoloid race. Padjadjaran Journal of Dentistry 2014;26(3).

8. Bajunaid SO, Baras B, Alhathlol N, Ghamdi AA. Evaluating the reliability of facial and hand measurements in determining the vertical dimension of occlusion. Int J Med Pharm 2017;5(1):1-1.

9. Akhma NE, Sumarsongko T, Rikmasari R. Correlation between the occlusal vertical dimension and the pupil rima oris distance among sundanese population. 
Padjadjaran Journal of Dentistry 2017;29(2).

10. Waseem-Ul-Ayoub D, Rashid R, Scholar PG. Novel Technique to Determine Vertical Dimension of Occlusion from Interpupillary Distance in Kashmiri Population. International Journal of Engineering Science 2017, 14955.

11. Misch CE. Objective vs. subjective methods for determining vertical dimensions of occlusion. Q International 2000;31:280-2.

12. Nagpal A, Parkash H, Bhargava A, Chittaranjan B. Reliability of different facial measurements for determination of vertical dimension of occlusion in edentulous using accepted facial dimensions recorded from dentulous subjects. The Journal of Indian Prosthodontic Society 2014;14(3):233-42.

13. McGee GF. Use of facial measurements in determining vertical dimension. The Journal of the American Dental Association 1947;35(5):342-50.

14. Pound E. The Mandibular Movements of Speech and Their Seven Related Values, JS Calif. St. Dent. Assoc 1966;34:435-41.

15. Oladipo GS, Esomonu C, Osogba IG. Craniofacial dimensions of Ijaw children and adolescents in Nigeria. $\mathbf{J}$ Biomed Int 2010;1(1):25-9.

16. Laestadius ND, Aase JM, Smith DW. Normal inner canthal and outer orbital dimensions. The Journal of pediatrics 1969;74(3):465-8. 